\title{
AN EXAMPLE IN THE THEORY OF HYPERCONTRACTIVE SEMIGROUPS
}

\author{
ANDRZEJ KORZENIOWSKI AND DANIEL W. STROOCK ${ }^{1}$
}

\begin{abstract}
Let $L=x\left(d^{2} / d x^{2}\right)+(1-x)(d / d x)$ on $C_{c}((0, \infty))$ be the Laguerre operator. It is shown that for $t>0$, and $1<p<q<\infty, e^{t l}: L^{p}\left(e^{-x} d x\right) \rightarrow$ $L^{q}\left(e^{-x} d x\right)$ has norm 1 if and only if $e^{-t} \leqslant(p-1) /(q-1)$ and the corresponding logarithmic Sobolev constant is not equal to $2 / \lambda$, where $\lambda$ is the smallest nonzero eigenvalue of $L$.
\end{abstract}

Let $(E, \mathscr{F}, m)$ be a probability space and $\left\{P_{t}: t>0\right\}$ a conservative Markov semigroup on $B(E)$ for which $m$ is a reversible measure (i.e. for each $t>0, P_{t}$ is symmetric on $\left.L^{2}(m)\right)$. Then, as an easy application of Jensen's inequality, $\left\|P_{t}\right\|_{L^{p}(m) \rightarrow L^{p}(m)} \leqslant 1$ for all $t>0$ and $p \in[1, \infty]$. In particular, each $P_{t}$ admits a unique extension $\bar{P}_{t}$ as a bounded operator on $L^{2}(m)$ and $\left\{\bar{P}_{t}: t>0\right\}$ is a semigroup of selfadjoint contractions. A well-studied example of this situation is the Ornstein-Uhlenbeck semigroup $\left\{\Gamma_{t}^{(d)}: t>0\right\}$ on $B\left(R^{d}\right): E=R^{d}, m(d x)=\gamma^{(d)}(d x)$ $=g^{(d)}(1, x) d x$, and $P_{t}=\Gamma_{t}^{(d)}$ is given by

$$
\Gamma_{t}^{(d)} f(x)=\int g^{(d)}\left(1-e^{-2 t}, y-e^{-t} x\right) f(y) d y
$$

where $g^{(d)}(\tau, \xi)=(2 \pi \tau)^{-d / 2} \exp \left(-|\xi|^{2} / 2 \tau\right), \quad(\tau, \xi) \in(0, \infty) \times R^{d}$. In connection with his work on constructive field theory, E. Nelson [2] discovered that $\left\{\Gamma_{t}^{(d)}\right.$ : $t>0$ \} enjoys a hypercontractivity property. Namely, he showed that for given $1<p<q<\infty,\left\|\Gamma_{t}^{(d)}\right\|_{L^{p}\left(\gamma^{(d)}\right) \rightarrow L^{q}\left(\gamma^{(d)}\right)} \leqslant 1$ if and only if $e^{-2 t} \leqslant(p-1) /(q-1)$. In addition, he noted that if $e^{-2 t}>(p-1) /(q-1)$, then $\left\|\Gamma_{t}^{(d)}\right\|_{L^{p}(\gamma) \rightarrow L^{q}(\gamma)}=\infty$. Since Nelson's initial discovery, many other examples of hypercontractive semigroups have been found (cf. F. Weissler [7, 8], F. Weissler and C. Mueller [9], and O. Rothaus [3-5]). In most cases the difficult part of the analysis lies in the attempt to obtain the optimal result (i.e. the smallest $T(p, q)>0$ such that $\left\|P_{t}\right\|_{L^{p}(m) \rightarrow L^{q}(m)} \leqslant 1$ for all $t \geqslant T(p, q))$. The work of L. Gross [1] shows that this question is closely related to that of finding the smallest $\alpha>0$ for which the logarithmic Sobolev inequality

$$
\int|f|^{2} \log |f|^{2} d m \leqslant \alpha \mathscr{E}(f, f)+\|f\|_{L^{2}(m)}^{2} \log \|f\|_{L^{2}(m)}^{2}
$$

Received by the editors March 27, 1984 and, in revised form, June 6, 1984. 1980 Mathematics Subject Classification. Primary 47D05; Secondary 46E30.

Key words and phrases. Laguerre semigroup, logarithmic Sobolev inequality, Ornstein-Uhlenbeck semigroup, hypercontractivity.

${ }^{1}$ The work of this author was supported in part by N.S.F. Grant MCS 8310542.

(C1985 American Mathematical Society $0002-9939 / 85 \$ 1.00+\$ .25$ per page 
holds, where $\mathscr{E}$ denotes the Dirichlet form associated with $\left\{\bar{P}_{t}: t>0\right\}$ (i.e.

$$
\mathscr{E}(f, f)=\sup _{t>0} \frac{1}{t}\left(f-\bar{P}_{t} f, f\right)_{L^{2}(m)}=\lim _{t \rightarrow 0} \frac{1}{t}\left(f-\bar{P}_{t} f, f\right)_{L^{2}(m)}
$$

and $\left.\operatorname{Dom}(\mathscr{E}) \equiv\left\{f \in L^{2}(m): \mathscr{E}(f, f)<\infty\right\}\right)$. Indeed, under mild conditions, Gross's analysis shows that (1) for a given $\alpha>0$ is equivalent to

$$
\left\|P_{t}\right\|_{L^{p}(m) \rightarrow L^{q}(m)} \leqslant 1, \quad e^{-4 t / \alpha} \leqslant \frac{p-1}{q-1} .
$$

(cf. D. Stroock [6, §9], for additional information). Further, Rothaus [3] has shown that the logarithmic Sobolev constant (i.e., the smallest $\alpha$ for which (1) holds) must be at least $2 / \lambda$, where

$$
\lambda=\inf \left\{\mathscr{E}(f, f):\|f\|_{L^{2}(m)}=1 \text { and } \int f d m=0\right\}
$$

is the gap between 0 and the rest of the spectrum of the generator $\left\{\bar{P}_{t}: t>0\right\}$. For the most part, the technique adopted for proving optimality has been to prove that (1) holds with $\alpha=2 / \lambda$ (cf. [9]).

The main purpose of this note is to provide a simple example for which the hypercontractivity constant is not $2 / \lambda$. To this end, take: $E=[0, \infty), m(d \rho)=$ $e^{-\rho} d \rho$, and for locally bounded measurable $f:[0, \infty) \rightarrow R^{1}$ having subexponential growth at $\infty$, define $P_{t} f$ so that

$$
P_{t} f\left(\rho^{2} / 2\right)=\left[\Gamma_{t / 2}^{(2)} \tilde{f}\right](\rho \omega), \quad t>0 \text { and } \rho \in[0, \infty),
$$

where $\tilde{f}(x)=f\left(|x|^{2} / 2\right), x \in R^{2}$, and $\omega=\left(\begin{array}{l}1 \\ 0\end{array}\right) \in R^{2}$. Then the following facts about $\left\{P_{t}: t>0\right\}$ are easy to check:

(i) $\left\{P_{t \mid B(E)}: t>0\right\}$ is a conservative Markov semigroup,

(ii) for each $t>0, P_{t}$ is symmetric on $L^{2}(m)$.

(6) Lemma. Let $1<p<q<\infty$ and $t>0$ be given. If $e^{-t} \leqslant(p-1) /(q-1)$, then $\left\|P_{t}\right\|_{L^{p}(m) \rightarrow L^{q}(m)} \leqslant 1$. If $e^{-t}>(p-1) /(q-1)$, then $\left\|P_{t}\right\|_{L^{p}(m) \rightarrow L^{q}(m)}=\infty$.

Proof. Note that for any $r \in[1, \infty)$ and any measurable $g:[0, \infty) \rightarrow R^{1},\|g\|_{L^{r}(m)}$ $=\|\tilde{g}\|_{L^{r}\left(\gamma^{(2)}\right)}$. Also, observe that for any locally bounded $f:[0, \infty) \rightarrow R^{1}$ having subexponential growth at $\infty, \Gamma_{t / 2}^{(2)} \tilde{f}=\widetilde{P_{t} f}, t>0$. Thus, $\left\|P_{t}\right\|_{L^{p}(m) \rightarrow L^{q}(m)} \leqslant 1$ is equivalent to $\left\|\Gamma_{t / 2}^{(2)} \tilde{f}\right\|_{L^{q}\left(\gamma^{(2)}\right)} \leqslant\|\tilde{f}\|_{L^{p}\left(\gamma^{(2)}\right)}$ for all locally bounded measurable $f$ : $[0, \infty) \rightarrow R^{1}$ which have subexponential growth at $\infty$. In particular, by Nelson's inequality, $\left\|P_{t}\right\|_{L^{p}(m) \rightarrow L^{q}(m)} \leqslant 1$ if $e^{-t} \leqslant(p-1) /(q-1)$. To prove that $\left\|P_{t}\right\|_{L^{p}(m) \rightarrow L^{p}(m)}=\infty$ if $e^{-t}>(p-1) /(q-1)$, consider the functions $f_{\lambda}(\rho)=$ $\exp \left(2^{1 / 2} \lambda \rho^{1 / 2}-\lambda^{2} / 2\right)$ for $\lambda>0$. In view of the preceding considerations, we need only check that

$$
\lim _{\lambda \rightarrow \infty}\left\|\Gamma_{t / 2}^{(2)} \tilde{f}_{\lambda}\right\|_{L^{q}\left(\gamma^{(2)}\right)} /\left\|\tilde{f}_{\lambda}\right\|_{L^{p}\left(\gamma^{(2)}\right)}=\infty
$$


when $(p-1) /(q-1)>e^{-t}$. By straightforward computation, one can easily see that

$$
\begin{aligned}
{\left[(\pi / 2)^{1 / 2} r \lambda\right]^{1 / r} } & \exp \left(\lambda^{2}(r-1) / 2\right) \\
& \leqslant\left\|\tilde{f}_{\lambda}\right\|_{L^{\prime}\left(\gamma^{(2)}\right)} \leqslant\left(1+(2 \pi)^{1 / 2} r \lambda\right)^{1 / r} \exp \left(\lambda^{2}(r-1) / 2\right)
\end{aligned}
$$

for any $\lambda>0$ and $r \in(1, \infty)$. At the same time,

$$
\left[\Gamma_{t / 2}^{(2)} \tilde{f}_{\lambda}\right](x) \geqslant \sup _{\theta \in S^{1}}\left[\Gamma_{t / 2}^{(2)} g_{\lambda \theta}\right](x)=\sup _{\theta \in S^{1}} g_{\lambda e^{-t / 2} \theta}(x)=\tilde{f}_{\lambda e^{-t / 2}}(x)
$$

where $g_{\eta}(x)=\exp \left(\eta \cdot x-|\eta|^{2} / 2\right)$ for $\eta \in R^{2}$ and we have used the fact that $\Gamma_{s}^{(2)} g_{\eta}=g_{e^{-s} \eta}$ for all $s>0$ and $\eta \in R^{2}$. After combining these, one easily arrives at the desired conclusion. Q.E.D.

To complete our analysis, we must compute the $\lambda$ associated with $\left\{\bar{P}_{t}: t>0\right\}$. To this end, let $\left\{Y_{n}: n>0\right\}$ be the normalized Laguerre polynomials (i.e. the normalized orthogonal polynomials on $[0, \infty)$ with respect to $m$ ) and define $H=\Delta-x \nabla$ on $C^{\infty}\left(R^{2}\right)$. Then, as is well known,

$$
\rho \frac{d^{2} Y_{n}}{d \rho^{2}}(\rho)+(1-\rho) \frac{d Y_{n}}{d \rho}(\rho)=-n Y_{n}(\rho), \quad n \geqslant 0 \text { and } \rho \in[0, \infty) \text {. }
$$

From this, it is an easy matter to check that

$$
H \tilde{Y}_{n}=-2 n \tilde{Y}_{n}, \quad n \geqslant 0 .
$$

Since $\Gamma_{t}^{(2)} f-f=\int_{0}^{t} \Gamma_{s}^{(2)} H f d s, t>0$, for all polynomials $f: R^{2} \rightarrow R^{1}$, we conclude that

$$
\Gamma_{t / 2}^{(2)} \tilde{Y}_{n}=e^{-n t} \tilde{Y}_{n}
$$

and therefore that

$$
P_{t} Y_{n}=e^{-n t} Y_{n}
$$

for all $t>0$ and $n \geqslant 0$. As an immediate consequence, we now have that

$$
\bar{P}_{t} f=\sum_{0}^{\infty} e^{-n t}\left(f, Y_{n}\right)_{L^{2}(m)} Y_{n}, \quad t>0 \text { and } f \in L^{2}(m) .
$$

In particular, the Dirichlet form $\mathscr{E}$ for $\left\{\bar{P}_{t}: t>0\right\}$ is given by

$$
\mathscr{E}(f, f)=\sum_{1}^{\infty} n\left(f, Y_{n}\right)_{L^{2}(m)}^{2}, \quad f \in L^{2}(m),
$$

and so the corresponding gap $\lambda$ is 1 .

By combining Gross's analysis, Lemma (6) and the preceding, we now have the following result.

(7) TheOREM. Let $m(d \rho)=e^{-\rho} d \rho$ on $[0, \infty)$ and define $P_{t}, t>0$, by (4). Then $\left\{P_{t}\right.$ : $t>0\}$ is a conservative Markov semigroup which is symmetric in $L^{2}(m)$. Let $\left\{\bar{P}_{t}\right.$ : $t>0\}$ be the semigroup of $L^{2}(m)$-selfadjoint contractions determined by $\left\{P_{t}: t>0\right\}$ and denote by $\mathscr{E}$ the associated Dirichlet form. Then

$$
1=\inf \left\{\mathscr{E}(f, f): f \in L^{2}(m),\|f\|_{L^{2}(m)}=1 \text { and } \int f d m=0\right\},
$$

On the other hand, the logarithmic Sobolev constant for $\mathscr{E}$ (i.e. the smallest $\alpha$ for which (2) holds) is 4. 
Remark. The semigroup $\left\{P_{t}: t>0\right\}$ in Theorem (7) can be described directly in terms of the Laguerre operator

$$
L=\rho \frac{d^{2}}{d \rho^{2}}+(1-\rho) \frac{d}{d \rho} \quad \text { on } C_{c}^{\infty}((0, \infty))
$$

Indeed, $\left\{P_{t}: t>0\right\}$ is the unique conservative Markov semigroup on $B((0, \infty))$ such that

$$
P_{t} f-f=\int_{0}^{t} P_{s} L f d s, \quad t \geqslant 0,
$$

for all $f \in C_{c}^{\infty}((0, \infty))$. Thus there are several reasons for calling $\left\{P_{t}: t>0\right\}$ the Laguerre semigroup. In this connection it is natural to suspect that the reason why, in this example, the logarithmic Sobolev constant $\alpha_{0}$ and the spectral gap $\lambda$ do not satisfy $\alpha_{0}=2 / \lambda$ may have something to do with the way in which $L$ degenerates at 0 .

\section{REFERENCES}

1. L. Gross, Logarithmic Sobolev inequalities, Amer. J. Math. 97 (1975), 1061-1083.

2. E. Nelson, Probability theory and Euclidean field theory (G. Velo and A. Wightman, editors), Lecture Notes in Physics, vol. 25, Springer, Berlin and New York, 1974, pp. 94-124.

3. O. S. Rothaus, Logarithmic Sobolev inequalities and the spectrum of Sturm-Liouville operators, J. Funct. Anal. 39 (1980), 42-56.

4. __ Logarithmic Sobolev inequalities and the spectrum of Schrodinger operators, J. Funct. Anal. 42 (1981), 110-120

5. Diffusion on compact Riemannian manifolds and logarithmic Sobolev inequalities, J. Funct. Anal. 42 (1981), 102-109.

6. D. W. Stroock, Introduction to the theory of large deviations, Springer-Verlag, Berlin and New York, 1984.

7. F. Weissler, Two-point inequalities, the Hermite semigroup and the Gauss-Weierstrauss semigroup, J. Funct. Anal. 32 (1979), 102-121.

8. Logarithmic Sobolev inequalities and hypercontractive estimates on the circle, J. Funct. Anal. 37 (1980), 218-234.

9. F. Weissler and C. E. Mueller, Hypercontractivity for the heat semigroup for ultraspherical polynomials and on the n-sphere, J. Funct. Anal. 48 (1982), 252-283.

Department of Mathematics, University of TeXas, Arlington, Texas 76019

Department of Mathematics, University of Colorado, Boulder, Colorado 80209

Current address (D. W. Stroock): Department of Mathematics, Massachusetts Institute of Technology, Cambridge, Massachusetts 02139 